\title{
The Smart Triad : Big Data Analytics, Cloud Computing and Internet of Things to shape the Smart Home, Smart City, Smart Business \& Smart Country
}

\author{
D.Radhika, D. Aruna Kumari
}

\begin{abstract}
Big data analytics, cloud computing \& internet of things are a smart triad which have started shaping our future towards smart home, city, business, country. Internet of things is a convergence of intelligent networks, electronic devices, and cloud computing. The source of big data at different connected electronic devices is stored on cloud server for analytics. Cloud provides the readymade infrastructure, remote processing power to consumers of internet of things. Cloud computing also gives device manufacturers and service providers access to "advanced analytics and monitoring", "communication between services and devices", "user privacy and security". This paper, presents an overview of internet of things, role of cloud computing \& big data analytics towards IoT. In this paper IoT enabled automatic irrigation system is proposed that saves data over "ThingSpeak" database an IoT analytics platform through ESP8266 wifi module. This paper also summarizes the application areas and discusses the challenges of IoT.
\end{abstract}

Index Terms - Internet of Things (IoT), Cloud Computing, Fog Computing, Reference IoT Layered Architecture (RILA), Big Data Analytics, Big data, Intelligent Networks

\section{INTRODUCTION}

The world in and around is running with busy schedules at work, and home. Time is more important when compared to money and effort put in to solve a problem. The common thing in most of us is we are connected through a smart phone. Since a decade working hard is replaced with being and working smart. In a hospital just few minutes without power supply might give trouble to the patient's life. In a farm, to water the crop as per the level of moisture in the soil is a challenge. The demonetization of Rupees 500, Rupees 1000 on November 8th 2016 in India pushed us to move from cash based payment to electronic payments. Educating ,training common man to use electronic payments is a challenge. Smart phones usage to be encouraged for daily transactions, bill payments, fund

Revised Version Manuscript Received on 16 September, 2019.

D.Radhika, Research Scholar, Computer Science Engineering, K L University, Guntur, AP, India.radhikarajasekhar@yahoo.com

Dr D.Aruna Kumari, Professor, Department ECM, K L University, Guntur, AP, India. aruna_d@kluniversity.in transfers etc. Road traffic monitoring and control is a very common problem to metropolitan cities during school, college and office timings.

To address problems with constraints like time, distance, money, human effort, the best solution is to connect the electronic devices using intelligent networks and store and analyze data. This convolution of big data analytics, cloud computing and internet of things in this era will drive us towards living in smart home, work at smart office, live in smart city, country and even do smart business.

Cloud computing is a internet based computing paradigm that believes in sharing computing resources in the form of infrastructure, data, platform as a service based on the demand of the user with pay per use fashion. Big data refers to data getting recorded at various electronic devices with properties such as huge volume, huge speed i.e. velocity, different kinds of data i.e. variety, data flow inconsistencies i.e variability, different formats, speed, correlated, connected i.e complexity and ultimate property is the knowledge from that data i.e value. CISCO prevision states that in the year 2008, the number of objects which are connected are more than human beings living on earth. It also predicted that by 2020 the number of things connected may cross 50 billion [1]

The remainder of the paper is structured as follows. Section II overview on IoT. Section III throws role of cloud computing and big data towards IoT. Section IV provides review of application areas of IoT. Section V Experiment and Results and Section VI concludes the paper besides providing directions for future work.

\section{OVERVIEW ON INTERNET OF THINGS}

\section{A. What is IoT?}

Internet of things is a virtual connection between things, people, animals, processes and almost everything around us. It can narrate a scenario where everything in and around our environment is made capable of automatically communicating with each other without any inter-human or human-to-machine interaction.

Heterogeneous group of industries, companies are working towards success of IoT. The companies from various sectors should agree upon a common standardized architecture for enabling communication between all these diverse "Things". 
It is easy to claim standardization in research environment but challenging to achieve in the real world. Reference architectures are of importance for standardization, as they list out the guidelines that are to be referred for planning the implementation of an IoT system [2].

\section{B. Terminology to be aware of:}

Thing: it is an instance of our surroundings or environment. Examples are car, house, fridge etc.

Device: It is part of a thing and is a sensor, actuator or tag.

Thing processes the devices based on context information and sends selected information to other objects based on the use case. Thing passes actions to actuators [2].

\section{Foreseeable IoT Components In IoT Reference Architecture:}

- Devices and things require interoperability and integration components.

- Techniques to define a context and action, and goal definition by rule engines known as context aware computing techniques.

- Complete architecture would require security guidelines.

\section{Basic Building Blocks of IoT Architecture}

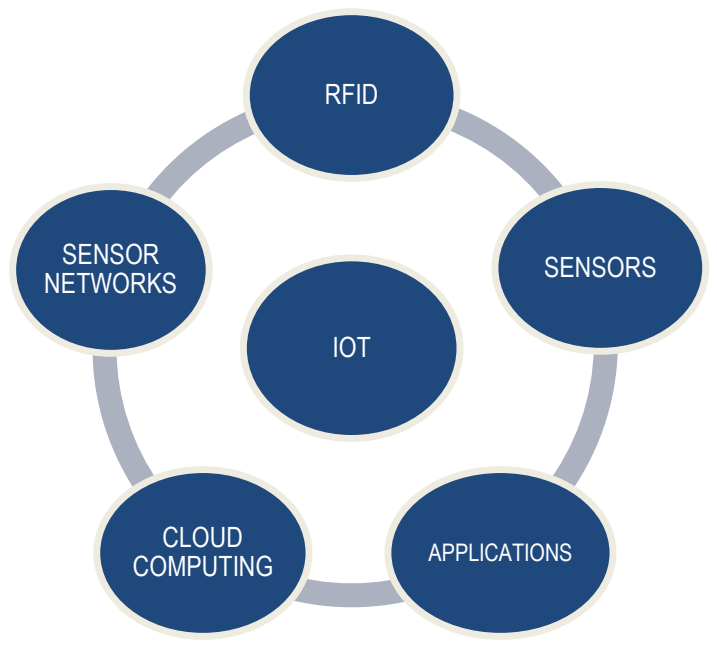

Fig.1 Basic Building Blocks of IoT

The Fig. 1 represents the basic building blocks of internet of things. It includes RFID, Sensors, Applications, Cloud Computing, Sensor Network.

- RFID: It is abbreviation for radio frequency identification which is an important basic building block of IoT. It uses electromagnetic fields to automatically identify and even track tags attached to objects.

- Sensor: Object that detects events or changes in its environment and sends the information to the computer which then tells the actuator (output devices) to provide the corresponding output is termed as Sensor. When ideal sensors not used the several deviations like noise, drift, hysteresis, digitization errors and so on are observed. They can be of various types like medical sensors, non medical sensors, chemical sensors, biosensors etc.

- Applications: These are an important building block that states in which scenarios we can implement IoT. The application scenarios include monitoring and control, information sharing and collaboration, big data and data analytics and many more.

- Cloud computing: It is one of the important building block and backbone of IoT. It is an internet based computing that works around the principle of pay per use fashion and service oriented architecture. It facilitates IoT users by providing "Software as a Service (SaaS)", "Platform as a Service (PaaS)", "Infrastructure as a Service (IaaS)".

- Sensor Network: An interesting building blocks where a group of tiny, typically battery-powered devices and wireless infrastructure that monitors and records conditions in any number of environments. There can be wired or wireless sensor networks.

\section{E. Architecture of IoT}

Reference IoT Layered Architecture, short "RILA" is a combination of things, devices and the user as shown in Fig.2. The functionalities of each layer in RILA is briefly given below, as detailed description of it cannot be accommodated in the context of this paper. The outcome of one layer is an input to another layer.

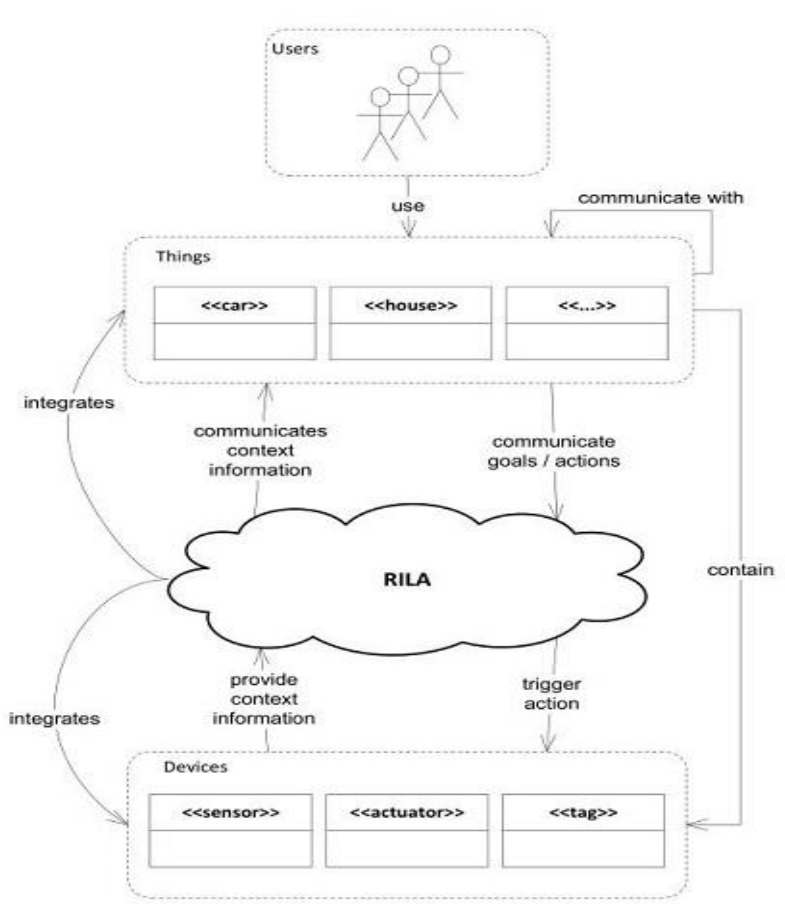

Fig.2 RILA (Reference IoT Layered Architecture)

In the Fig. 3 the RILA consists of 6 layers and two "crosssection-layers" namely "Security" and "Management" influence all other layers.

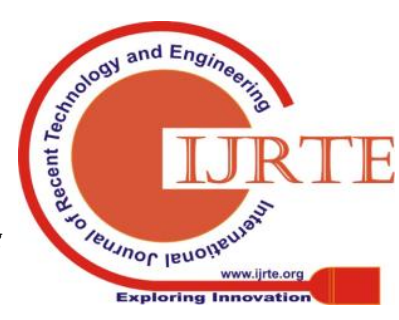




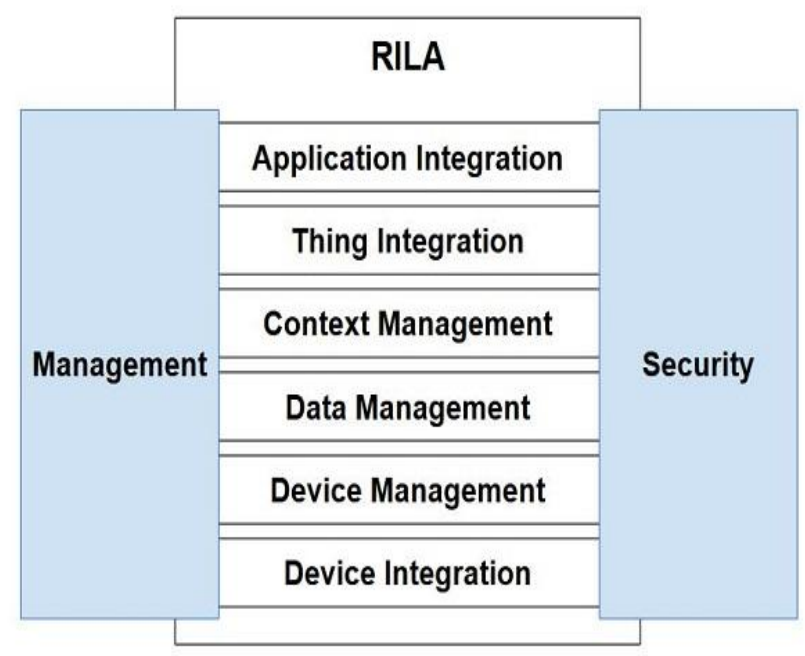

Fig.3 RILA 6 Layers \& 2 cross-section-layers

- Device Integration: This layer connects different varieties of devices, their measurements, actions at device level.

- Device Management: This layer collects measurements from device integration and verifies to confirm and translate the status change to the actuator. Based on the use case it may be implemented using a data warehouse or data farm.

- Context Management: This layer performs tasks such as goals of the thing, consume the context situation(s) of other things, produce the (own) context situation of the thing, evaluate the (own) context situation towards the goal, trigger actions that help to fulfill the goal according to the evaluated rules, publish context situations for other things.

- Thing Integration: This layer functionality is to find other things and communicate with them.

- Application Integration: This layer is a service layer or a simple user interface that connects the user to the thing and implementation is use case specific.

- Security, Management: The cross section layers security and management influence all other layers [3].

\section{F. Advantages of $I o T$}

A shift in technology has always shown pros and cons in its initial phases. The below is a list of practical advantages visible using IoT.

- Transportation: IoT introduced a monitory sensor that helps to track the distance and time locations and other contributing factors for tracking transportation of goods.

- Inventory Management: To track the location of products in real time IoT uses tag radio frequency sensors. They are instrumental in tracking the level of inventory, and to stock it in advance, by placing orders automatically, alerting on a need basis.
- Data Management: This layer holds data of a thing.

- Product Promotions: Frequent shoppers are intimated with product promotion details through mobiles using IoT. It can be implemented by keeping track of shopping interests, shopper history of physical shoppers and shoppers over the internet.

- Web User Intelligence: Using IoT customer buying pattern, preferences, interests, can be tracked by third party web data aggregators.

- Product Maintenance Services \& Warranty: IoT enables product tracking. It is useful when product is lost, require warranty or maintenance services.

- Enhanced Vending Technology: Machine to machine communication concept of IoT has enhanced the working of vending machines by enabling them to communicate to monitor inventory levels, determine pricing, etc.

The above listed are only few benefits of internet of things. It enhances the quality of life in all aspects leading to smart home, smart city, smart business, and smart country.

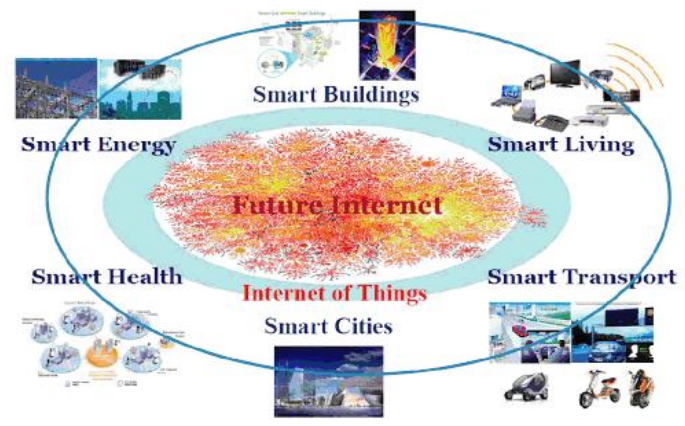

Fig.4 Smart World

\section{G. Disadvantages of IoT:}

There are few disadvantages of IoT that effect moral principles, human employment and reliability on technology.

- Ethical issues: In the Code of Ethics for the IEEE it states that it will strive "to treat fairly all persons regardless of such factors as race, religion, gender, disability, age, or national origin" (IEEE, 2012). Certain group of people may not have internet, no money to purchase devices, technology to support IoT. The Code of Ethics for ACM says it will "respect the privacy of others" and "honor confidentiality" which might be broken by collecting information on people and their habits.

- As no system is robust and fault free it is not advisable for current generation to rely only on technology on a day to day basis, to make decisions based on the information that it gives.

- People who are less educated or who are not trained to use technology are prone to loss of jobs. 


\section{H. Challenges with IoT:}

Using IoT systems a number of security and privacy issues will arise as they are ubiquitous and pervasive. The IoT application must be economical, efficient, credible with effective security and privacy. The other challenges with IoT are

- Sensing a complex environment requires innovative methods to sense and deliver information from physical world to the cloud.

- Connectivity may be through wired or wireless standards based on applications.

- Power is critical as certain IoT applications may run for years over batteries and reduce overall energy consumption.

- Security is very important in protecting user privacy and manufacturers IP, detecting and blocking malicious activity.

- IoT application development is complex and not simple.

- Availability of cloud services is vital for IoT applications as they require end-to-end solutions [11].

\section{ROLE OF CLOUD COMPUTING AND BIG DATA ANALYTICS TOWARDS IOT}

a) Big Data

The word data is replaced with big data exhibiting characteristics volume, variety, velocity, veracity, validity, volatility.

- Volume: Enormous volume of data is generated by machines, network and human interaction on systems like social media.

- Variety: Data is available in the form of PDFs, photos, emails, videos, monitoring devices, audio, etc. This refers to variety. As there are many types and sources of data. Mining and analyzing such unstructured data creates problems.

- Velocity: The pace with which the data flows in real time. Analytics at real time can help researchers and businesses make valuable decisions that provide strategic competitive advantages and ROI.

- Veracity: The dirty data, noises, abnormalities in data getting accumulated in system refers to veracity. There should be continuous procedures to prevent and even clean dirty data.

- Validity: Is the data accurate and correct for intended use refers to validity. It is main key for taking correct and timely decision.

- Volatility: In organizations data gets accumulated over a period of time. Volatility refers to how long data is valid and how long it should be stored. Based on this, decision can be made about relevancy of data to the intended analysis [8].

b) Fog with IoT

The Paradigm driving the success of IoT is Fog Computing (FC). It is extension of Cloud Computing paradigm. The characteristics of FC are:

- Low latency and location awareness

- Wide-spread geographical distribution

- Mobility

- Very large number of nodes
- Predominant role of wireless access

- Strong presence of streaming and real time applications

- Heterogeneity

The characteristics given above make the FC the appropriate platform for a number of critical IoT services and applications, namely, Connected Vehicle, Smart Grid, Smart Cities, Wireless Sensors and Actuators Networks (WSANs) [3].

\section{c) Big Data, Cloud Computing, Internet of Things}

The internet of things is collecting big data from users, things, devices using the basic architecture containing RFID, sensors, applications, cloud computing, sensor networks. Big data is collected stored and analyzed under the architecture of cloud computing. Big data analytics requires parallel processing power in distributed environment. Cloud computing provides services by pay per use fashion. The services provided by cloud are software as a service (Saas), platform as a service (Paas), infrastructure as a service (Iaas). The traditional measurement mechanisms, algorithms to analyze big data do not work well in terms of environment, cost, quality time and even reliability. If mining is performed by each or group of organizations with their own methodologies, it might incur lot of expenditure, redundancy of work, time, effort and cost. There is no guarantee of standardized procedures, expertise, reliability for the method followed for big data analytics at every business. Algorithms for big data analytics under specific domain, customized scenarios need to be standardized and made available as a service in the cloud framework. There by (Maas) mining as a service if incorporated under the framework of cloud will result in standardized, efficient, scalable algorithms for big data analytics. This will increase algorithms reliability, expertise, quality and decrease time, effort, cost to design the algorithm by organizations and businesses [9][10].

\section{REVIEW OF APPLICATION AREAS OF IOT}

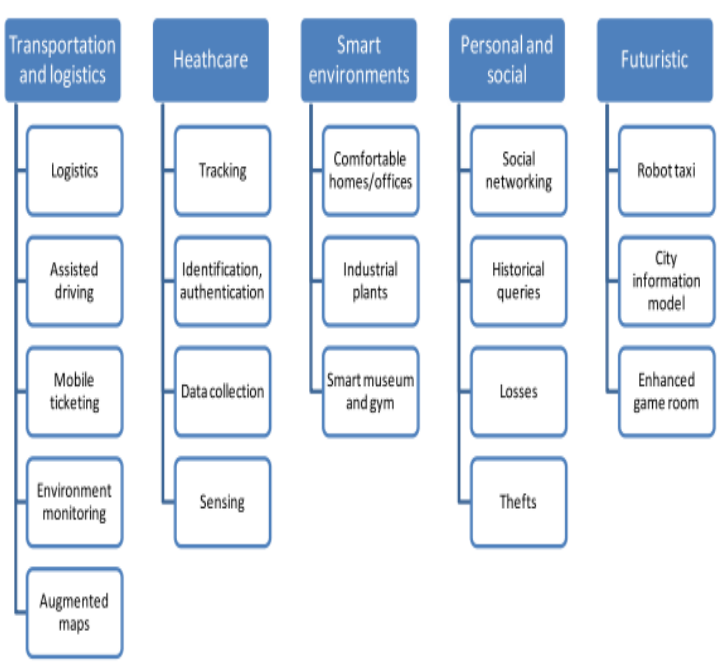

Fig.5 Domain wise classification of application areas of IoT

Published By:

Blue Eyes Intelligence Engineering 
The above Fig.5 shows domain wise classification of application areas of IoT. The brief description about them is as follows:

- Transportation and Logistics: IoT can be used to monitor and track logistics, machine guided driving of cars and automobiles. It can also be used to switch on or off street lights based on sunlight and darkness, empty the trash bins when full, manage traffic jams. Touristic maps are supported with tags that allow NFC-equipped phones to browse it and automatically call web services providing information about hotels, restaurants, monuments and events related to the area of interest for the user.

- Healthcare: IoT can be used to monitor health problems in people from different age groups, gender and needs. An alert can be sent to the guardian of the deceased or medical practitioner, or health centre, or doctor to provide support. For example monitoring the health of a person who underwent heart surgery, using a IoT enabled wearable device. This system can be used to monitor elders and disabled people.

- Smart Environment: IoT helps in detecting, monitoring emissions from factories and vehicles that would drive in minimizing air pollution. IoT can send warnings and alerts about earthquakes, tsunamis by detecting tremors and floods by monitoring water level of rivers and dams.

- Personal and Social: Applications based on IoT for fall detection are expected to provide security and help to people with disabilities or elders. The IoT integrated with assistive technology will improve the life of people with disabilities. The applications include text-voice converter, education aids for reading and writing, robotics for mobility, household maintenance, smoke detectors, remote lighting and water control. People with hearing disability and not aware of American Sign Language, hand talk is a less priced portable gesture-to-speech glove prototype.

- Futuristic: In future the driver less taxi will be replacing existing driver driven taxis. The taxi destination will be fixed by customer and on electronic payment and authorized access the taxi allows customers to board and transports them to the destination.

- IoT Enabled Farm Control: goal to deploy IoT enabled farm control to improve overall operational efficiency, feed management, security, and improvement in productivity. IoT Analytics and venue analytics can be used to improve overall farm management and making smart and precision farming a reality.

- Scenarios: electronic devices can identify your need and order goods, adjust temperature, switch on or off devices, safe guard or alert predicting medical problems [5].

\section{EXPERIMENT AND RESULTS}

The experiment developed for Smart Irrigation System is an IoT enabled device which is capable of automating the irrigation process by analyzing the moisture of soil. It provides water supply at the right time, in right quantity and at the right place in field which plays a vital role in the plant's growth. Water management remotely is also challenging task, especially the management becomes more difficult during the shortage of water, which may otherwise damage the crop.

By using sensors like moisture, rain, etc. water supply for irrigation can be managed easily by analyzing the condition of soil and climate. Soil moisture sensors smartly measure the soil moisture and based on that data, field is irrigated automatically with less human interventions.

The soil moisture sensor detects moisture levels in the soil and sends the moisture value to the Arduino. The Arduino microcontroller compares the received value with threshold value and if the value is less than threshold value then it switches on the pump through relay so that the plants can be irrigated automatically. This data is saved over "ThingSpeak" database through ESP8266 Wifi Module.

THINGSPEAK: ThingSpeak is an IoT analytics platform service that allows you to aggregate, visualize and analyze live data streams in the cloud. ThingSpeak provides instant visualizations of data posted by your devices to ThingSpeak. It is often used for prototyping and proof of concept IoT systems that require analytics and also to visualize sensor data in real-time.

WORKING: Sensors, or things, sense data and typically act locally. ThingSpeak enables sensors, instruments, and websites to send data to the cloud where it is stored in either a private or a public channel. ThingSpeak stores data in private channels by default, but public channels can be used to share data with others. Once data is in a ThingSpeak channel, you can analyze and visualize it, calculate new data, or interact with social media, web services, and other devices. The collected data can also be downloaded. It can be downloaded as required file types such as (.CSV, .XML) etc.

\section{IMPLEMENTATION AND RESULT ANALYSIS}

The experiment consists of output screens for uploading, searching, viewing and for storing of dataset through authenticated users such as admin or user. The dataset date and time wise is collected from the IoT device as shown in block diagram in fig 6 . The dataset through cloud is exported as soildataset.csv file. The dataset is analysed to generate day wise average, minimum, maximum moisture level reports. This analysis facilitates, guides in planning the frequency time intervals for watering the soil. 
The Smart Triad : Big Data Analytics, Cloud Computing and Internet of Things to shape the Smart Home, Smart City, Smart Business \& Smart Country

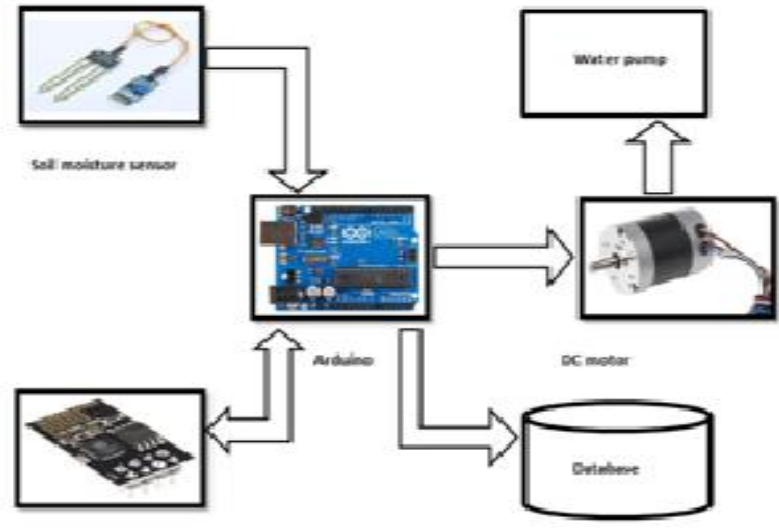

Fig 6. Block Diagram

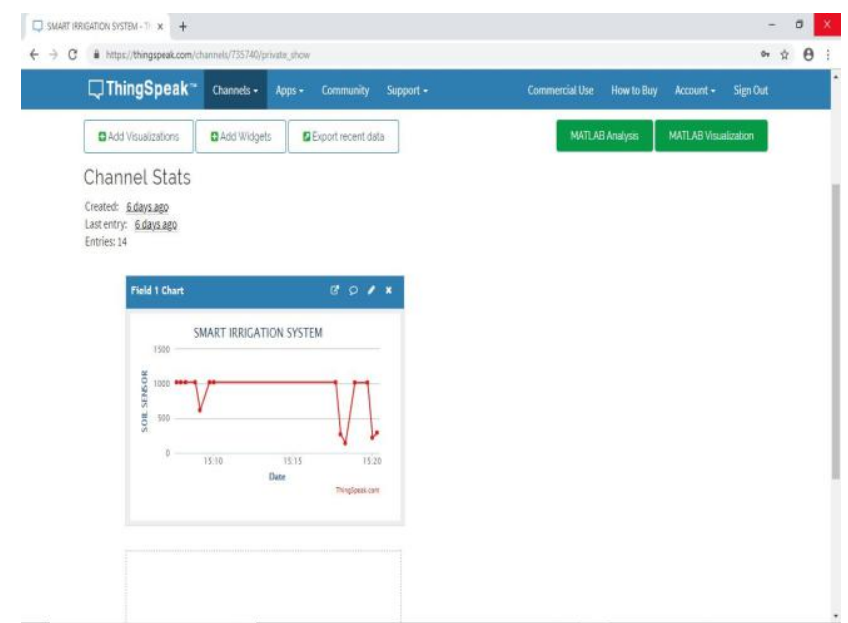

Fig 7: Testing

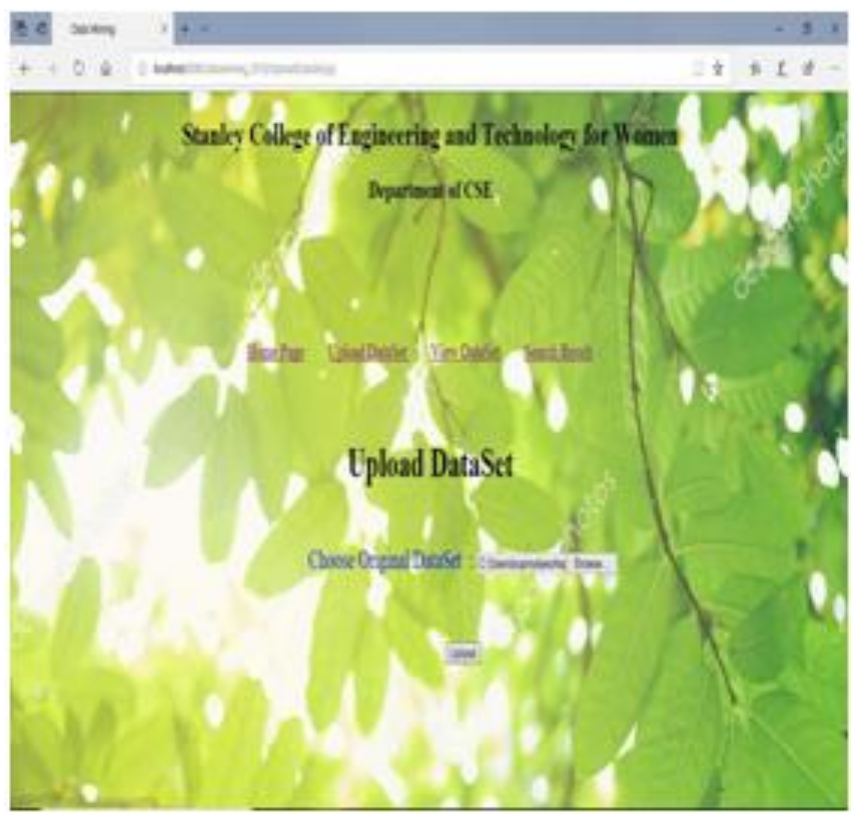

Fig 8:upload data

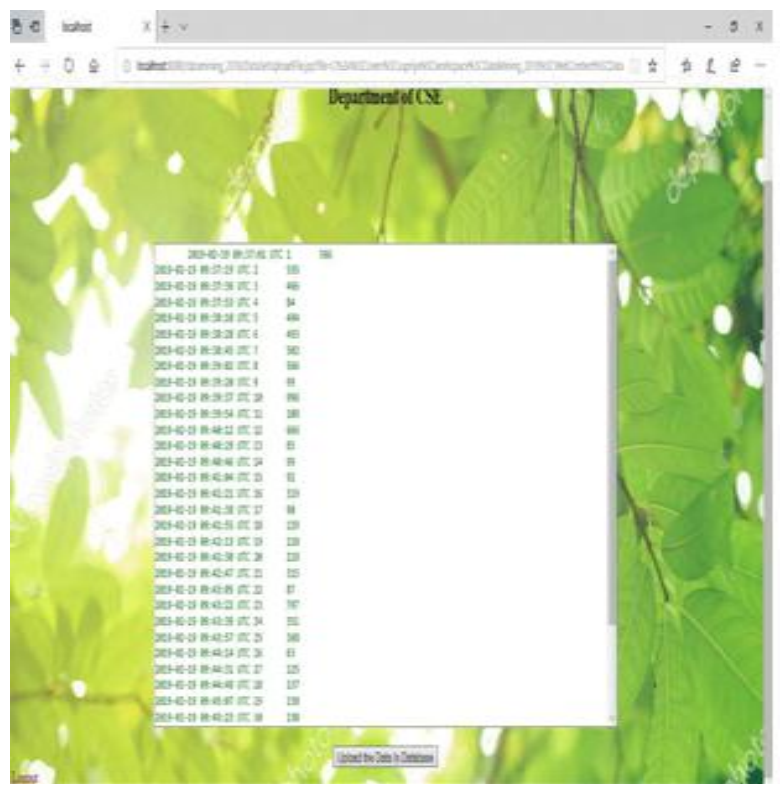

Fig 9. checking Data Set

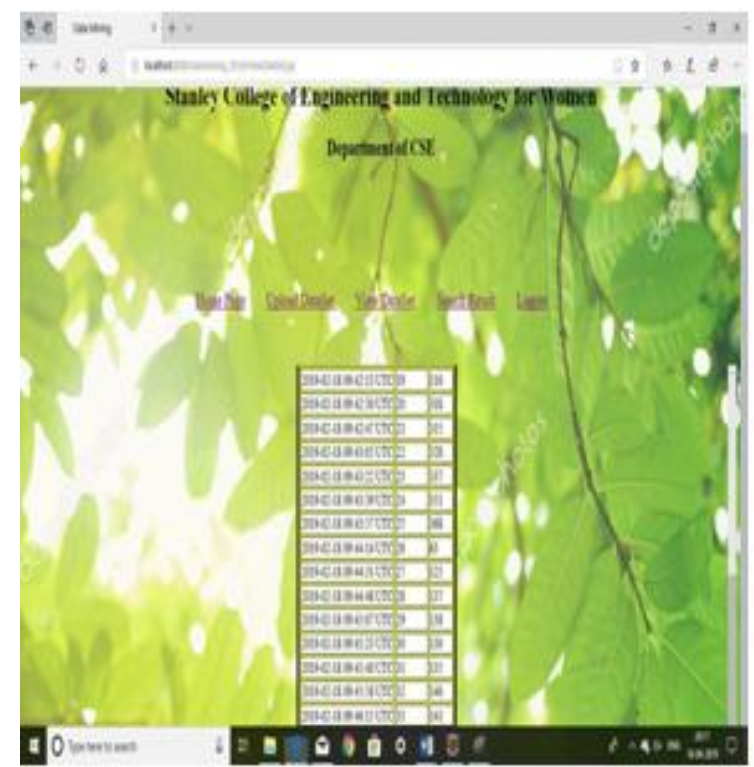

Fig 10. Uploaded Data Set

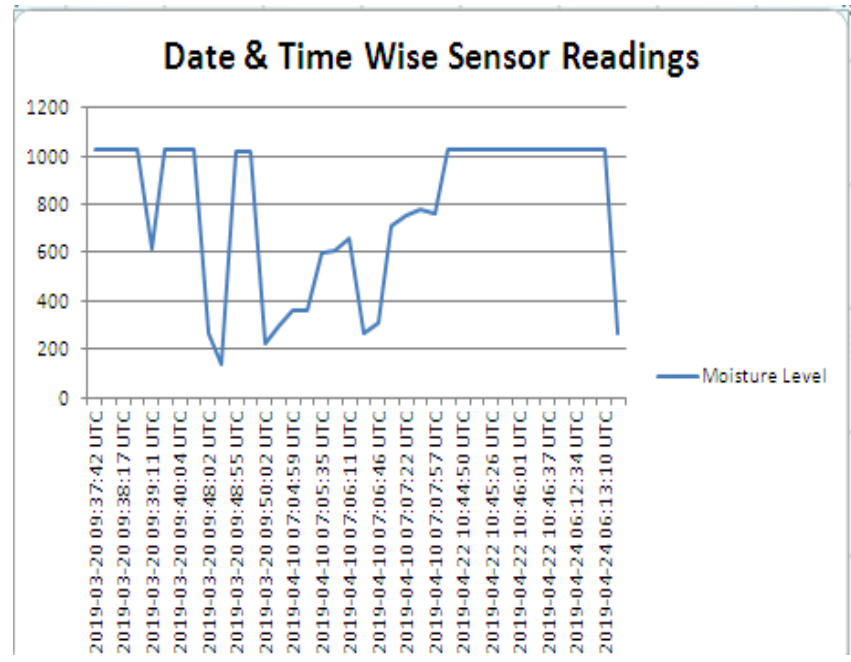

Fig 11. Sensor Readings Date and Time Wise

Published By:

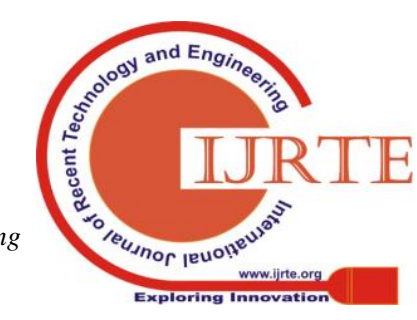




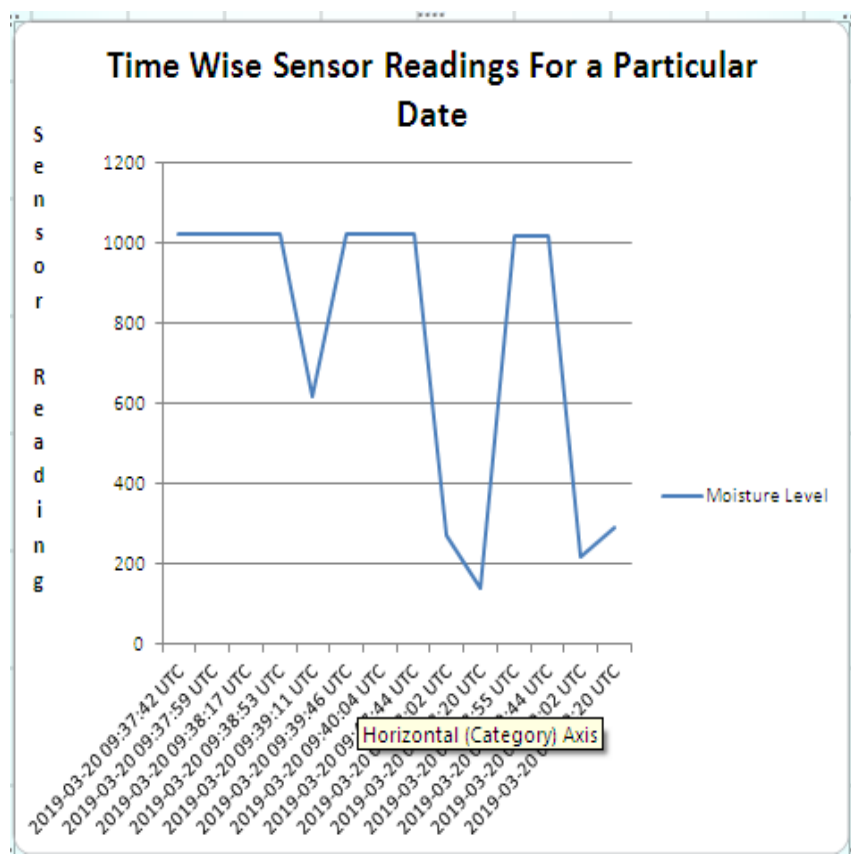

Fig 12. Time Wise Sensor Readings For a Particular Date

Discussion

The main challenge with IoT is its implementation. Using IoT systems a number of security and privacy issues will arise as they are ubiquitous and pervasive. The IoT application must be economical, efficient, credible using effective security and privacy. When ideal sensors not used the several deviations like noise, drift, hysteresis, digitization errors and so on are observed. Developing IoT applications is not simple and requires cloud services.

\section{CONCLUSION AND FUTURE WORK}

This paper focused on the need for connecting electronic devices enabled with services provided over cloud servers. It also described the overview of IoT, role of cloud computing and big data, towards success of IoT, the application areas of internet of things.

\section{REFERENCES}

[1] Evans, Dave. "The internet of things." How the Next Evolution of the Internet is Changing Everything, Whitepaper, Cisco Internet Business Solutions Group (IBSG) 1 (2011): 1-12.

[2] M. Abomhara and G. M. Køien, "Security and privacy in the Internet of Things: Current status and open issues," 2014 International Conference on Privacy and Security in Mobile Systems (PRISMS), Aalborg, 2014, pp. 1-8.

[3] F. Bonomi. Connected vehicles, the internet of things, and fog computing. VANET 2011, 2011.

[4] Lee, Hwa. "The Internet of Things and Assistive Technologies for People with Disabilities: Applications, Trends, and Issues." Internet of Things and Advanced Application in Healthcare (2016): 32.

[5] Atzori, Luigi, Antonio Iera, and Giacomo Morabito. "The internet of things: A survey." Computer networks 54.15 (2010): 2787-2805.

[6] Talia, Domenico. "Toward cloud-based big-data analytics." IEEE Computer Science (2013): 98-101.

[7] Demirkan, Haluk, and Dursun Delen. "Leveraging the capabilities of service-oriented decision support systems: Putting analytics and big data in cloud." Decision Support Systems 55.1 (2013): 412-421.

[8] Normandeau, Kevin. "Beyond volume, variety and velocity is the issue of big data veracity." Inside Big Data (2013).

[9] Demirkan, Haluk, and Dursun Delen. "Leveraging the capabilities of service-oriented decision support systems: Putting analytics and big data in cloud." Decision Support Systems 55.1 (2013): 412-421.
[10] Radhika, D., and D. Aruna Kumari. "A Framework for Exploring Algorithms for Big Data Mining." Indian Journal of Science and Technology 9.17 (2016).

[11] Chase, Jim. "The evolution of the internet of things." Texas Instruments(2013).

[12].http://www.circuitstoday.com/arduino-soil-moisture-sensor

[13].http://fab.cba.mit.edu/classes/863.14/tutorials/Programming/serialwifi. $\underline{\mathrm{html}}$

\section{AUTHORS PROFILE}

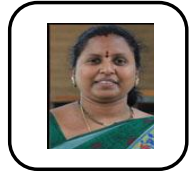

Mrs D Radhika is Asst Professor at Department of CSE, Stanley College of Engineering \& Technoogy for Women, Hyderabad, Telanagana, India. Data mining, Big Data Analytics, Internet of Things, Privacy of Big Data are the interested areas of research. She published 7 research articles in International Journals. She also presented and published research papers in National and International Conferences. She is currently pursuing research on "Privacy of Big Data".

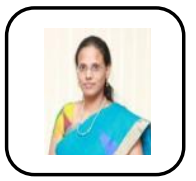

Dr Aruna Kumari Professor at Department of CSE,VJIT, Hyderabad, Telangana India. She is Fellow of CSI FCSI), and Fellow of IEEE (FIEEE). She is DST Young Scientist Awardee (Govt. of India). She is Ph.D Supervisor at Department of CSE KL-University, Guntur, Andhra Pradesh. Data mining, Cloud Computing, Privacy \& Security of Big Data, Big Data Analytics are her interested areas of research. She has more than 70 research articles in International Journals and Conferences. She is Currently working on research project "Design and Development of Effective Privacy Preserving Data Mining for Cardiac Cancer and Diabetic Health Care" funded by DST-SERB. She has organized several National and International Conferences and Workshops.

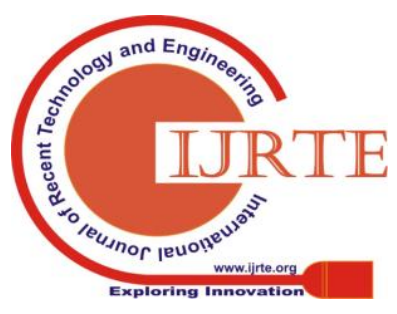

